\title{
Programa Autoestima: Uma ferramenta Web pública e amigável que integra formação de profissionais da saúde e acolhimento psicossocial da população
}

\author{
Maria Cristina M. Domingues ${ }^{1}$, Kelsey M. Melo ${ }^{1}$, Alexandre F. Miranda ${ }^{1}$, Alina \\ Zoqui de Freitas Cayres ${ }^{2}$, Rosangela Elias ${ }^{2}$ \\ ${ }^{1}$ Instituto de Pesquisas Tecnológicas do Estado de São Paulo, SP, 05508-901, Brazil \\ cmachado@ipt.br, kelsey@ipt.br, alexandrefm@ipt.br
}

\author{
${ }^{2}$ Secretaria de Saúde do Estado de São Paulo, SP, 05403-000, Brazil \\ azoqui@saude.sp.gov.br, relias@saude.sp.gov.br
}

\begin{abstract}
This article describes the implementation of a public digital web platform for the realization of courses for graduate professionals in the assistance area of the Unified Health System (SUS) and provide remote service to citizens through a public call center tool, developed by the Government of the state of São Paulo. The platform was developed with the objective of contributing in the context of the Covid 19 pandemic, offering spaces for listening and care for the population, in the virtual modality, given the need for people to remain in social isolation. The implementation of this digital tool brings two challenges, managing the schedules of health professionals and adopting a tool for mediation of the psychological care process. In the view of e-government, there is a barrier to digital inclusion and it is necessary to reflect on strategies that can expand access to the resource.
\end{abstract}

Resumo. Este artigo descreve a implementação de uma plataforma digital web pública para a realização de cursos destinados a profissionais da área assistencial do Sistema Único de Saúde (SUS) e atendimento remoto aos cidadãos por meio de uma ferramenta pública de teleatendimento, desenvolvida pelo Governo de São Paulo. A plataforma foi desenvolvida com o objetivo de contribuir no contexto da pandemia Covid19, ofertando espaços de escuta e cuidado para a população, na modalidade virtual, visto a necessidade de se manter em isolamento social. Do ponto de vista do governo eletrônico sinaliza que, por conta do perfil que tem utilizado o programa, existe a barreira da inclusão digital e se faz necessário refletir sobre estratégias que possam ampliar o acesso ao recurso.

\section{Introdução}

A pandemia por COVID-19 trouxe grandes desafios para a população em geral e afetou drasticamente a saúde em todo o mundo (Kadir,2020). O elevado número de contaminados e de óbitos, na casa de milhões de pessoas no mundo e de centenas de milhares no Brasil, impacta na saúde mental, com distintas manifestações de sofrimento psíquico e impactos de médio e longo prazo decorrentes dos efeitos dessa ampla crise de dimensão sanitária, social, política e econômica (Opas, 2020; Iasc, 2020; Fiocruz, 2020).

A prolongada situação de isolamento social, os efeitos diretos e indiretos dos lutos de familiares e próximos decorrentes da COVID-19, sem a possibilidade de se ritualizar esse momento de despedida, associado a um contexto amplo de crise global, gera aumento nas queixas e sintomas relacionados à saúde mental (Opas, 2020; Iasc, 2020; Fiocruz, 2020). 
Surge a demanda, de um lado, por novas ofertas de espaços de escuta e cuidado para a população como consequência deste contexto e, de outro lado, a busca por adaptação e formação de profissionais de saúde do Sistema Único de Saúde (SUS) para a adoção de novas estratégias e técnicas de atendimentos na modalidade virtual.

Diferentes órgãos e setores do Governo do Estado de São Paulo, mobilizados por essas necessidades, uniram-se a Secretaria de Estado da Saúde de São Paulo, entre eles o Fundo Social de Solidariedade, o Instituto de Pesquisas Tecnológicas do Estado de São Paulo IPT, a Universidade Virtual do Estado de São Paulo - UNIVESP, a Companhia de Processamento de Dados do Estado de São Paulo - PRODESP, o Instituto de Psicologia da Universidade de São Paulo - IP/USP, o Serviço Brasileiro de Apoio às Micro e Pequenas Empresas - Sebrae SP e o Conselho de Secretários Municipais de Saúde - COSEMS para criar o Programa Autoestima.

$\mathrm{Na}$ perspectica do ideário do governo eletrônico, essas parcerias se constituíram por meio da identificação e participação de assessorias técnicas oriundas de diversos setores da sociedade que se somaram para construir este espaço de forma coletiva e solidária, por meio da doação de parcela de seu tempo de trabalho e expertise técnica (Cordeiro et al, 2012; Castro, 2018; Pereira e Bordin, 2020; Carvalho e Rover, s.d.; Carvalho e Silva, 2020; Naskar et al, 2020). Com isso, tal iniciativa desenvolveu-se o portal para ofertar conteúdos sobre saúde mental e atendimento on-line para acolhimento psicossocial da população, valorizando o protagonismo do cidadão na busca por saúde mental, a qualificação para as equipes do SUS ao cuidado em saúde mental, além de um espaço onde os serviços possam compartilhar suas ações e experiências de forma dinâmica, ampliando o repertório e diversificando as práticas de cuidado e promoção em saúde mental, com ênfase nas modalidades virtuais. Com foco na tríade técnica-performativa dos profissionais participantes dos cursos, por meio da formação (aulas), atendimento (em estágio prático) e supervisão dos casos atendidos com corpo docente para a transmissão desses conhecimentos.

O Programa Autoestima, conforme descrito no portal, visa formação à distância e apoio psicossocial para a Rede de Atenção Psicossocial - RAPS dos municípios paulistas, através da implantação de uma Plataforma Digital (www.autoestima.sp.gov.br) para constituição e acesso de acervo com conteúdos relevantes, ofertando conhecimento e tecnologias inovadoras, desenvolvendo processos formativos visando o melhor atendimento à população em sofrimento psíquico, além de orientações aos que possuem necessidades de tratamento e cuidado específico em saúde mental, decorrentes de situações de estresse e/ou crise humanitária.

O artigo está organizado da seguinte forma: na seção 2, a discussões e concepção da plataforma; na seção 3, a implementação do projeto; na seção 4, o agendamento das consultas; na seção 5, os aspectos técnicos do teleatendimento; na seção 6 , estágio atual do projeto; na seção 7, a conclusão e trabalhos futuros.

\section{Discussões e concepção da plataforma}

O desenvolvimento de uma aplicação Web é um processo interdisciplinar, utilizando técnicas de design e de desenvolvimento de software. Devido à separação e evolução dos vários contextos de aplicação destas áreas, tem sido possível observar a implementação de várias abordagens no seu processo de criação e desenvolvimento (Gothelf 2017). No projeto da Plataforma Autoestima, por ter várias entidades envolvidas com visões e diferentes anseios, fez-se necessário a utilização de algumas ferramentas para a discussão, 
concepção e desenvolvimento, são elas: Design Thinking, Experiência do Usuário (UX) e Métodos Ágeis para o desenvolvimento e entregas.

Para Gothelf (2017), Design Thinking permite determinar e modelar aquilo que está sendo proposto pelos stakeholders. O Design Thinking traz em seu nome a palavra design, mas a sua aplicação não é exclusiva ao design, mas "uma forma de resolver problemas através da criatividade" (IDEO, s.d.). Também conhecido como tempestade de ideias, é defendido que se deve abordar os problemas com uma mentalidade de principiante, centrado no usuário, aberto e mantendo a empatia, otimismo, interação, criatividade e ambiguidade. É uma abordagem bastante aberta que aplica o seguinte método: descoberta inicial sobre o que as pessoas realmente precisam; seguindo a criação de múltiplas ideias e hipóteses através de protótipos; e culminando com a realização de testes, de forma a aprender mais sobre o problema e o usuário que interage, fazendo repetidamente para melhorar ou divergir para novas ideias e hipóteses. Para Norman (2013) é uma fase em que se deve questionar tudo e abranger todos os atores envolvidos no projeto. O Design Thinking do projeto foi feito em reuniões semanais com os stakeholders (web designer, desenvolvedores, psicólogos, educadores, profissionais da saúde, equipe de marketing, e gerente do projeto) utilizando artefatos como atas de reuniões, mapas mentais, modelo de processo e protótipos.

Após o Design Thinking o foco do projeto foi na experiência do usuário (UX), que de acordo com Norman e Nielsen (The Definition of User Experience (UX), s.d.) abrange todos os aspetos da interação do usuário final com a Plataforma. A UX cobre todos os aspectos de como as pessoas utilizam a plataforma: como se sentem quando acessam, qual a sensação no uso das funcionalidades, o quão bem se adequa ao seu propósito e se encaixa no contexto geral em que a utilizam. Algumas perguntas foram feitas para os envolvidos no projeto, são elas: Como será utilizada? Qual o perfil de quem vai utilizar a ferramenta? Com que tipo de ferramenta os usuários estão acostumados? Como vai interagir com a plataforma? Posteriormente a esse levantamento foram desenhadas as interfaces, as funcionalidades e os objetos da plataforma. Só após o desenho da interface é que foi entregue à equipe de desenvolvimento que utilizou a metodologia ágil para implementar a solução e gerenciar as entregas.

A metodologia Ágil, de acordo com Beck, et al., 2001, nasce no seio da área do desenvolvimento de software com a necessidade de dar uma resposta mais adaptada às expectativas de custo, tempo de entrega das demandas e da qualidade do produto. Expectativas estas que no processo cascata são mais frustradas por serem baseadas estritamente em abordagens mais rígidas e lineares.

A prioridade no projeto foi a satisfação dos clientes, no caso a Secretaria de Saúde, o governo do estado e, sobretudo, a população usuária do sistema (o cidadão comum e o profissional de saúde) através de entregas rápidas e contínuas; outro ponto importante a relatar foi a aceitação de novos requisitos numa fase tardia do ciclo de desenvolvimento; também, a recorrência de períodos de entrega de poucas semanas, dando preferência a períodos ainda mais curtos; e, por fim, a homologação regular, fazendo os ajustes e adaptações necessárias. Com todo esse cenário, foi essencial adotar a metodologia de desenvolvimento ágil, que para Kumar, Sharma \& Gupta (2016) são métodos essenciais para validar a satisfação do cliente por meio das entregas, facilitando o acompanhamento e ajudando a equipe de desenvolvimento a demonstrar as soluções tecnológicas adotadas nas diferentes ações e etapas do projeto. 


\section{Implementação do projeto}

A equipe de Web Design entregou para a equipe de desenvolvimento de software o CSS (Cascading Style Sheets), uma folha de estilo composta por camadas utilizada para definir a apresentação (aparência) em páginas da internet que adotam para o seu desenvolvimento linguagens de marcação HTML, com toda a identidade visual, a definição do perfil dos usuários e os diversos cenários de interação.

A equipe de desenvolvimento, em posse das páginas e dos artefatos produzidos no Design Thinking, fez a modelagem do banco de dados e da arquitetura do sistema, levando em consideração a abrangência do programa de governo. O modelo de banco de dados possui 41 tabelas, sendo 25 dedicadas aos cadastros dos usuários (com 2 diferentes perfis: cidadão e profissional de saúde), 6 para a publicação de Notícias, 5 para a inscrição e publicação de cursos, 3 para o agendamento de atendimentos, e 2 para condições dos cadastros.

Para o desenvolvimento do ambiente proposto foram utilizadas tecnologias voltadas para a área de desenvolvimento Web. A princípio, foi definido que a estrutura da plataforma deveria abrigar conteúdos informacionais, como textos e mídias, de fácil utilização e manutenção para que a equipe da Secretaria da Saúde e da Psicologia da USP pudessem posteriormente alimentá-los e mantê-los. Para isso, analisou-se a possibilidade de criar um sistema de gerenciamento de conteúdo com o objetivo principal de auxiliar na inserção de conteúdos, imagens, vídeos, entre outros. Para a implementação da interface e das funcionalidades, foi necessária a utilização das linguagens de programação C\#, HTML, Javascript, JQuery, CSS, Bootstrap e tecnologias responsivas junto ao gerenciador de banco de dados SQL Server.

Para a infraestrutura de produção foram alocados quatro servidores virtuais, sendo dois servidores de aplicação e dois servidores de banco de dados. Para homologação da plataforma foram utilizados dois servidores, um para aplicação e outro para o banco de dados. Este último tem como finalidade a execução dos testes de homologação e aprovação, pela Secretaria do Estado da Saúde, na procura de inconsistências de funcionalidades existentes e em novas que são agregadas à plataforma durante o desenvolvimento. Feita a aprovação, a plataforma é atualizada, estando disponível para o público.

Os servidores de aplicação utilizam o sistema operacional Windows Server 2019, edição Datacenter, versão de 64 bits, que contém o IIS (Internet Information Services), que é utilizado para o gerenciamento e acesso via internet à plataforma. As configurações do servidor são de 2 GB de RAM, 70 GB de armazenamento e com o processador Intel Xeon de $2.40 \mathrm{GHz}$. Os servidores de banco de dados utilizam PaaS collocation com o Microsoft SQL Server 2019. Toda a infraestrutura de hospedagem do projeto foi disponibilizada pela PRODESP.

O processo do Programa Autoestima é bem simples. Para quem entra na plataforma parece um site de produção de informações de saúde, mas quando é realizado o cadastro, tanto pela população quanto pelo profissional da saúde, entra em um sistema, com perfis distintos. O profissional da saúde pode fazer cursos de aperfeiçoamento e realizar atendimentos; para tanto, é necessário fazer o gerenciamento de uma agenda e, no dia e hora do atendimento, entrar na ferramenta de teleatendimento. A população, em geral, pode consumir conteúdos do site e, se cadastrada e autenticada, pode solicitar atendimento por meio do agendamento e, depois, acessar, no dia e horário do atendimento, a ferramenta online de teleatendimento para acolhimento psicossocial breve. 
Um dos requisitos do projeto era de que o agendamento fosse simples e intuitivo. Esse objetivo foi alcançado com o desenvolvimento de funcionalidades, ao invés de utilizar plugins já existentes (componente pronto).

\section{Teleatendimento e escolha da ferramenta}

Segundo Santos Júnior e Monteiro (2020), nesse momento de pandemia e de adaptação do processo de ensino aprendizagem, as ferramentas de videoconferências constituem um recurso síncrono eficaz para interação a distância e com grandes potencialidades pedagógicas. Além de um meio de comunicação, seja para trabalho, como para uso pessoal. A evolução dessas ferramentas facilitou a comunicação e popularizou o serviço de modo que surgiram diversas opções no mercado. A equipe do projeto analisou quatro ferramentas para o uso como meio de atendimento, Microsoft Teams, Google Meet, Zoom e BigBlueButton.

Os critérios utilizados para a escolha de uma ferramenta adequada são de interface simples, menor quantidade de etapas e cliques para realizar uma chamada, criação de link único e restrito para os participantes, possibilidade de acessar sem obter cadastro ou instalação e suporte à integração, com foco na experiência do usuário, minimizando barreiras de acesso, comuns à população que busca o uso de recursos tecnológicos.

A ferramenta de videochamadas Zoom, foi uma das que mais se popularizou, pela sua facilidade de criação de conta, instalação e criação de reuniões, porém nem toda a população tem conhecimento básico de computação, e isso se torna um obstáculo para a utilização da ferramenta, pois é necessária a instalação do aplicativo seja no computador ou em dispositivos móveis e realizar um cadastro. Além disso, seria necessário aporte financeiro para custear assinatura que viabilizasse o uso a mais de um usuário simultâneo e por período maior de tempo ( 1 hora a 1hora e meia para atendimento individual e até 3 horas em atendimento grupal).

O Google Meet e o Microsoft Teams são ótimas ferramentas que apresentam diversos recursos, porém ambos necessitam de cadastro para acesso à chamada. No Microsoft Teams é necessário um participante cadastrado para que permita o acesso de outros participantes, além de sugerir a instalação do aplicativo ou extensão no navegador antes do acesso à chamada, o que pode dificultar o uso.

O BigBlueButton é uma ferramenta de código aberto, ou seja, gratuita e disponibilizada para uso próprio, não depende da disponibilidade do serviço de uma empresa e é possível habilitar e gerenciar as chamadas em uma infraestrutura própria, o que foi feito pela Secretaria do Estado da Saúde. Com uma interface simples, é possível acessar a chamada sem muita dificuldade e sem a necessidade de um cadastro, apesar de não ter tantos recursos como as outras ferramentas já citadas, não há a necessidade das mesmas, uma vez que é apenas utilizada a funcionalidade de chamada por vídeo. Porém, por ser uma ferramenta que utiliza recursos atuais, não é compatível com alguns aparelhos antigos, principalmente os portáteis. Algo que também pode acontecer com as demais ferramentas em teste.

Analisando as características das diferentes soluções analisadas na Tabela 1 foi escolhida a solução BigBlueButton, pela facilidade de uso e por atender as necessidades dos usuários. 
Tabela 1 - Mapa comparativo das ferramentas utilizadas para Teleatendimento

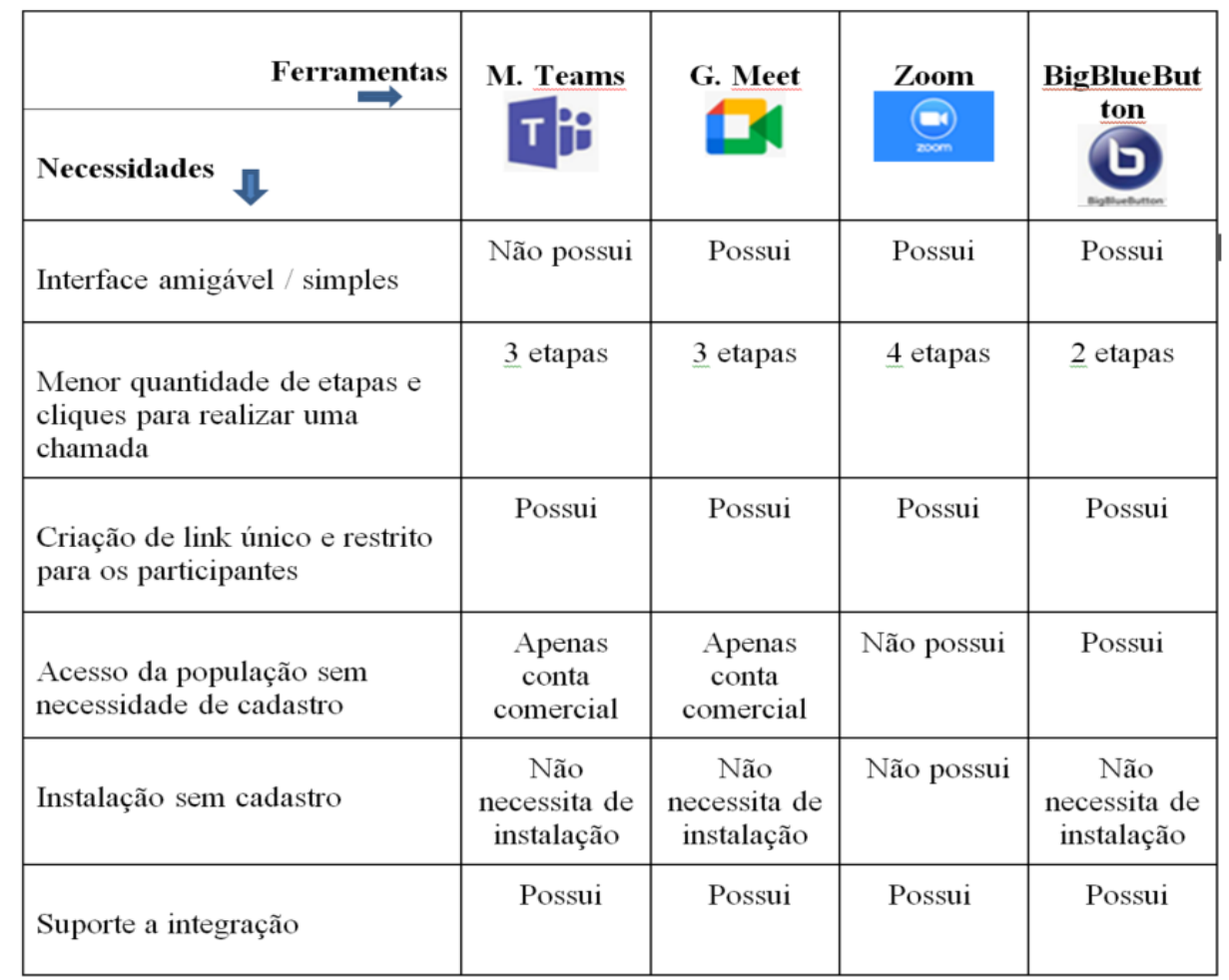

\section{Estágio atual do portal e do programa}

O portal foi implementado com sucesso, e disponibilizado para o público em janeiro de 2021, já tendo mais de 2.000 usuários cadastrados, 8.800 acessos/mês, disponibilizados 15 cursos para profissionais de saúde do SUS, em duas edições, e realizou cerca de 1.000 atendimentos virtuais realizados por meio de aproximadamente 300 profissionais em 3 meses.

Quanto ao perfil dos usuários cadastrados, a maioria é composta por mulheres $(80,0 \%)$, brancas $(60,0 \%)$ e pardas $(23,0 \%)$, mais jovens (de 21 até 40 anos, 63,4\%) com nível de escolaridade elevado (ensino superior incompleto até pós-graduação soma-se $67,3 \%$ do total) e residentes da região metropolitana de São Paulo (61,2\%).

Esse perfil pode sinalizar um dos principais desafios do programa, relacionado as dificuldades que parcela da população, principalmente de faixa etária elevada e com menor escolaridade (possivelmente também menor renda, embora essa informação não tenha sido incluída no perfil), apresenta dificuldades do ponto de vista do acesso, uso e manuseio de ferramenta digital, apesar da popularização dos recursos eletrônicos e digitais.

Por outro lado, o uso dessa ferramenta por esse perfil, de pessoas mais jovens e economicamente ativas e que, pelo momento de vida que se encontram, nem sempre buscam por um atendimento em saúde mental, principalmente pela via institucional e presencial, está promovendo o acesso de um público que geralmente fica mais distante da administração pública e do SUS, por procurar com menos frequência serviços públicos. 


\section{Conclusão e trabalhos futuros}

O portal do Autoestima tem servido como um auxílio para a população que procura por atendimento e acolhimento psicossocial breve, além de ser utilizada por diversos profissionais para formação através da realização de cursos teórico-práticos. A ferramenta de vídeo chamada escolhida vem se mostrando eficiente durante sua utilização. Apesar da incompatibilidade de alguns aparelhos, pode ser facilmente contornado por outra solução temporária como, por exemplo, o WhatsApp.

Para melhorar o uso e a experiência da população usuária do portal, pretende-se fazer a criação automática das salas de atendimento ao ser agendada uma consulta, disponibilizando o link no momento do atendimento, assim como melhorias e padronização visual, tanto para computador quanto para dispositivos móveis.

Contudo, na perspectiva do ideário do governo eletrônico de se incentivar uma relação cada vez mais acessível e democratizada à população em geral, os dados sinalizam que ainda se faz necessário discutir o problema da inclusão digital. Refletir sobre possíveis estratégias que possam viabilizar uma maior cobertura do programa para outros públicos que enfrentam tal dificuldade, que cria essa barreira de acesso a oferta de um cuidado em saúde mental que poderia beneficiá-los. Criar espaços colaborativos de uso de recursos tecnológicos e algumas bases de acesso presenciais em serviços de saúde localizados em alguns centros urbanos principalmente no interior do estado, como disponibilização de computador, tablete ou celular para atendimento virtual ao público, poderia auxiliar na ampliação dessa cobertura.

Cabe ressaltar que a oferta de teleatendimentos era menos incentivada e desenvolvida, com certo tensionamento de entidades de classe, como conselhos profissionais de medicina, psicologia, e outros, recurso que, com o advento da pandemia COVID-19, mostrou-se viável e muito potente. Com isso, incentivos e investimentos, sem tantas resistências, são recentes e tem se voltado para o desenvolvimento e o aprimoramento de tecnologias que possam viabilizar esse recurso.

\section{Referências}

Beck, K., Beedle, M., Bennekum, A. v., Cockburn, A., Cunningham, W., Fowler, M., Thomas, D. (2001). Manifesto for Agile Software Development. Obtido em https://agilemanifesto.org/

Castro, R.V.F. (2018) Governo eletrônico e cooperação interorganizacional: potencialidades, desafios e controvérsias da política de implantação de uma solução de processo administrativo. Monografia de conclusão de curso. Universidade de Brasilia, Departamento de Gestão de Políticas Públicas, 2018, 104p. (https://bdm.unb.br/bitstream/10483/22283/1/2018_RafaelVieiraFernandesDeCastro.pdf )

Carvalho, MA; Rover, A. A abordagem da Interação Humano-Computador na formação das Comunidades Virtuais de Prática dos Ambientes Virtuais Colaborativos de e-Gov Brasil. Programa de Pós-Graduação em Engenharia e Gestão do Conhecimento Universidade Federal de Santa Catarina, Florianópolis - SC, Brasil. (https://40jaiio.sadio.org.ar/sites/default/files/T2011/SSI/879.pdf)

Carvalho, H.; Silva, C. E-gov no Brasil e os desafios da pandemia de Covid-19. Revista $\begin{array}{llllll}\text { Inteligência Empresarial. } & \text { V. 42, N. } & \text { Nol. } & 2020\end{array}$ (https://inteligenciaempresarial.emnuvens.com.br/rie) 
Cordeiro, A. et al. (2012) Governo eletrônico e redes sociais: informação, participação e interação. RECIIS - Revista Eletrônica de Comunicação, Informação e Inovação em Saúde, Rio de Janeiro, v. 6, n. 2, p. 1-8, jun. (https://www.reciis.icict.fiocruz.br/index.php/reciis/article/view/588)

Gothelf, J. (2017). Lean vs. Agile vs. Design Thinking. Sense \& Respond Press.

IDEO. (s.d.). IDEO Design Thinking. Obtido de https://designthinking.ideo.com/

Fundação Oswaldo Cruz (Fiocruz). Recomendações e orientações em saúde mental e atenção psicossocial na COVID-19 / Rio de Janeiro: Fiocruz, 2020. 342 p.

Kadir, M. A. (2020). "Role of telemedicine in healthcare during COVID19 pandemic in developing countries" Telehealth and Medicine Today. Obtido em https:/telehealthandmedicinetoday.com/index.php/journal/article/download/187/279?inl ine $=1$.

Inter-Agency Referral Guidance Note for Mental Health and Psychosocial Support in Emergency Settings (IASC) Como lidar com os aspectos psicossociais e de saúde mental referentes ao surto de COVID-19. IASC, 2020.

Naskar, J. et al. SEHAT: Systematic Egovernance for Healthcare for All with Technology. Geneva Challenge, 2020. (https://www.researchgate.net/publication/343530257_SEHAT_Systematic_Egovernance_for_Healthcare_for_All_with_Technology)

Norman, D. (2013). The Design of Everyday Things (Revised and Expanded Edition ed.). London: MIT Press.

Norman, D., \& Nielsen, J. (s.d.). The Definition of User Experience (UX). Obtido em: https://www.nngroup.com/articles/definition-user-experience/

Organização Pan-Americana da Saúde (OPAS). Manejo Clínico de Condições Mentais, Neurológicas e por Uso de Substâncias em Emergências Humanitárias. Guia de Intervenção Humanitária mhGAP (GIH-mhGAP). Brasilia: Organização PanAmericana da Saúde; 2020.

Pereira, T.O.; Bordin, R.. Governo Eletrônico: O Uso De Tecnologias Da Informação Na Gestão Em Saúde De Novo Hamburgo (RS). In: Abdala, PRZ e Bordin, R. Gestão Pública: casos, análises e práticas, p.235-246, 2020

Santos Junior, V. B., Monteiro, J. C. S. (2020). Educação e Covid-19: as tecnologias digitais mediando a aprendizagem em tempos de pandemia. Revista Encantar - Educação, Cultura e Sociedade. Obtido em: https://revistas.uneb.br/index.php/encantar/issue/view/455 